\title{
Epitope-tagging of the endogenous murine BiP/GRP78/Hspa5 locus allows direct analysis of the BiP interactome and protein misfolding in vivo.
}

\author{
Yunqian Peng ${ }^{1 \#}$, Zhouji Chen $^{1 \#}$, Peter Arvan², and Randal J. Kaufman ${ }^{1 *}$
}

${ }^{1}$ Degenerative Diseases Program, Sanford-Burnham-Prebys Medical Discovery Institute, 10901 N. Torrey Pines Rd., La Jolla, CA 92037

${ }^{2}$ Division of Metabolism, Endocrinology \& Diabetes, the University of Michigan Medical School, Ann Arbor, MI 48105, USA

\# Equal Contributions

* Corresponding author: rkaufman@sbpdiscovery.org 


\section{ABSTRACT}

BiP/GRP78, encoded by the Hspa5 gene, is the major HSP70 family member in the endoplasmic reticulum (ER) lumen, and controls ER protein folding. BiP's essential functions in facilitating proper protein folding are mainly mediated through its dynamic interaction with unfolded or misfolded client proteins, and by serving as a negative regulator of the Unfolded Protein Response. A mechanistic understanding of the dynamics of BiP interaction with its protein partners is essential to understand ER biology, and therefore, we have sought to develop a tractable model to study misfolded protein interaction with BiP. For this purpose, we have used homologous recombination to insert a 3xFLAG epitope tag into the endogenous murine Hspa5 gene, just upstream from the essential KDEL signal necessary for ER localization of BiP. Tagging $\mathrm{BiP}$ in this way did not alter Hspa5 expression under basal or ER-stress induced conditions in hepatocytes ex vivo or in fibroblasts. Furthermore, the tag did not alter the cellular localization of $\mathrm{BiP}$ or its functionality. All of these findings in primary tissue culture were also confirmed in vivo in livers of heterozygous mice with one WT and one FLAG-tagged Hspa5 allele. Hepatocyte-specific BiP-FLAG modification did not alter liver function or UPR signaling. Importantly, immunoprecipitation with anti-FLAG antibody completely pulled down FLAG-tagged BiP from lysates of BiP-FLAG expressing livers. In summary, we generated a novel model that can be used to investigate the $\mathrm{BiP}$ interactome in vivo under physiological and pathophysiological conditions in a cell type-specific manner. This tool has the capability, for the first time, to provide an unbiased approach to identify unfolded and misfolded BiP-client proteins, and to provide new information on the role of $\mathrm{BiP}$ in many essential ER processes. 


\section{INTRODUCTION}

Protein misfolding is a protein-specific error-prone process in all cells. In particular, $\sim 30 \%$ of all cellular proteins are directed into the endoplasmic reticulum (ER). Protein folding in the ER is challenging because it requires many chaperones and catalysts to assist folding and prevent aggregation in a densely packed unfavorable environment comprised of oxidizing conditions, fluctuating $\mathrm{Ca}^{2+}$ concentrations and requiring both proper disulfide bond formation and posttranslational modifications(1). Significantly, only proteins that achieve their appropriate 3dimensional structures can traffic to the Golgi apparatus because of an exquisitely sensitive mechanism that identifies misfolded proteins and retains them in the ER for further productive protein folding or targets them to the degradation machinery mediated by the cytosolic $26 \mathrm{~S}$ proteasome or macroautophagy. Protein trafficking in the ER is guided by the addition, trimming and modification of asparagine-linked core oligosaccharides in order to engage lectin-based folding machinery for proper protein triage (1). Significantly, accumulation of misfolded proteins in the ER initiates adaptive signaling through the unfolded protein response (UPR), a tripartite signal transduction pathway that transmits information about the protein folding status in the ER to the nucleus and cytosol to restore ER homeostasis(2, 3). If the UPR cannot resolve protein misfolding, cells may initiate cell death pathways. Stress induced by accumulation of unfolded or misfolded proteins in the ER is a salient feature of differentiated secretory cells and is observed in many human diseases including genetic diseases, cancer, diabetes, obesity, inflammation and neurodegeneration. To elucidate the fundamental etiology of these diseases it is essential to identify which proteins misfold in response to different stimuli, with a future therapeutic goal to learn how to intervene to prevent misfolding.

The characterization of protein misfolding in vivo under different physiological conditions is limited due to the absence of conformation-specific antibodies, which are available for some viral glycoproteins, but are mostly lacking for endogenous cellular proteins. In addition, there is a need for an unbiased approach to identify the full spectrum of unfolded and misfolded proteins in the $E R$, in order to uncover the extent of misfolding of different protein species during disease progression, as well as the impact of different stimuli that can exacerbate ER protein misfolding. The most reliable surrogate for the misfolding of ER client proteins is their interaction with the "Binding Protein" known as BiP (encoded by HSPA5) which is a heat-shock protein 70 ER chaperone exhibiting peptide-dependent ATPase. BiP was originally characterized as a protein 
that binds immunoglobulin heavy chains to maintain them in a folding-competent state prior to their oligomerization with light chains (4). It was also recognized that glucose-deprivation induces a set of genes encoding glucose-regulated proteins, the most abundant being the ER protein GRP78, which is identical to BiP (5). Further analysis demonstrated that BiP expression is induced by protein misfolding in the ER through activation of the UPR.

Intriguingly, increased BiP levels feed-back to negatively regulate further UPR activation. One hypothesis posits that BiP binding to the UPR sensors IRE1, ATF6 and PERK inhibits their signaling (6), although there is no direct evidence to support this notion in physiological settings in vivo. Early studies to analyze protein misfolding demonstrated that only misfolded proteins that bind BiP activate the UPR and those that do not bind BiP do not activate the UPR (7-13). Unfortunately, however, there are no BiP antibodies currently available that can efficiently recognize BiP-client protein complexes in the absence of chemical crosslinkers, thus limiting the ability to study protein misfolding in the ER. As BiP provides many essential ER functions (including regulating Sec61 for co-translational and post-translational translocation into the ER, protein folding and degradation, maintenance of $\mathrm{ER} \mathrm{Ca}^{2+}$ stores, repressing UPR signaling, etc.), characterizing BiP interactions in vivo is essential to understand all these processes, and will provide significant insight into the role of ER protein misfolding in disease pathogenesis.

BiP IP from whole tissue lysates has the limitation that BiP is ubiquitously expressed; thus, IP recovers BiP and its partner proteins from multiple cell types. With this in mind, we have sought the ability to follow cell type-specific BiP interactions at different stages of disease progression. In addition, we wanted to avoid BiP overexpression, because this increases non-physiological BiP interactions (10). Therefore, we used homologous recombination to generate a conditional allele in mice with insertion of a 3xFLAG tag into the C- terminus of the endogenous BiP (Hspa5) coding sequence, just upstream from the KDEL ER localization signal. The engineered allele is designed such that upon cell type-specific Cre-induced deletion, expression of BiP-3xFLAG from the endogenous locus will permit endogenous BiP expression with the ability to identify BiPinteractors by anti-FLAG IP.

\section{MATERIALS AND METHODS}

\section{Generation of BiP-3xFlag mice.}


We generated a conditional knock-in mouse model by modifying of the Hspa5 locus. This was achieved by floxing a targeted WT exon 9-pA cassette upstream of the knock-in exon 9 where a 3XFLAG sequence was introduced immediately prior to the KDEL ER retention signal. Additionally, a FRT-flanked neomycin cassette was introduced into the floxed region. The genetic modification was introduced into Bruce4 C57BL/6 ES cells (14) via gene targeting. Correctly targeted ES cell clones were identified and then injected into goGermline blastocysts $(15,16)$. Male goGermline mice were bred to $\mathrm{C} 57 \mathrm{BL} / 6$ females to establish heterozygous germline offspring on a C57BL/6 background.

\section{a. Vector construction.}

A replacement vector targeting Hspa5 exon 9 coding sequence region (CDS region) was generated by assembly of $4(A B C D)$ fragments using sequential cloning. The first fragment which encompassed the 3 kb 5'-homology arm was generated by PCR amplified from C57BL/6 genomic DNA using primers P2093_41 and P2093_51. The second and third fragments which comprise loxP-exon9-BGHpA and exon 9-3xFlag were synthesized by Genewiz, respectively. The fourth fragment comprising the 3.2kb 3'-homology arm was generated by PCR amplified from C57BL/6 genomic DNA using primers P2093_44 and P2093_54. Synthesized fragments and PCR primers used to amplify the fragments included all the restriction enzyme sites required to join them together and to ligate them into the Surf2 vector backbone (Ozgene). The final targeting vector 2093_Teak_ABCD contained a FRT-flanked neomycin selection (neo) cassette, an exon 9 coding sequence sequentially with an inserted bovine growth hormone $(\mathrm{BGH})$ polyA tail, an additional exon 9 coding sequence sequentially with a 3xFLAG tag cassette right before the KEDL sequence, 5' and 3'-loxP site (Figure 1A). For sequence information of the primers see Suppl Table 1. The targeting vector was entirely sequenced and then linearized by digestion with Pmel before electroporation into C57BL/6 Bruce4 ES cells (14). Neo-resistant ES cell clones were screened by qPCR to identify potentially targeted clones.

\section{b. Targeting murine ES cells through homologous recombination.}

TaqMan® copy number reference assays were used to measure copy number in the genome. Two pairs of primers were used to amplify the WT locus at the extreme 5' and 3' positions to detect 2 copies from the WT allele and 1 copy from the targeted allele (primers, 2093_Lo5WT and 2093_LoWT3). Another primer pair targeting Neo sequence was used to test the targeted allele (primer, 1638_goNoz). Two genes from $Y$ chromosome (1 copy) and chromosome 8 (2 
copy) were used as control. Two positive clones, Clones I_1D08 and I_1G08, were confirmed as correctly targeted and were used to injection into goGermline blastocysts.

\section{c. Production of mice heterozygous for a BiP-FLAG allele.}

ES cells from clones I_1D08 and I_1G08 were injected into goGermline donor blastocysts to generate chimeras. A total of 84 injected blastocysts were transferred into 7 recipient hosts. These resulted in 35 offspring, of which 28 were male chimeras. Four males were chosen for mating with homozygous Flp mice. A total of 17 pups was born from three litters, including 10 WT and 7 WT/conKI (Figure 1B).

\section{Isolation and culture of primary hepatocytes and skin fibroblasts.}

Mouse primary hepatocytes were isolated by portal vein perfusion of collagenase as described (17). Murine skin fibroblasts were prepared by collagenase (Type II and Type IV, Sigma) digestion of abdominal skins dissected from a female BiP-FLAG-Heterozygous (Het) mouse and an Hspa5 wild type littermate (6-weeks old). The primary hepatocytes and skin fibroblasts were cultured in DMEM/10\% FBS. After overnight culture, cells were transduced with Ad- $\beta$ Gal or Ad-Cre at an $\mathrm{MOI}$ of 34 . Where specified, cells were treated with castanospermine (CST) or tunicamycin (Tm) to induce ER stress.

\section{Mouse experiments.}

Four female BiP-FLAG-Het mice and 4 of their female littermates were used for an in vivo experiment. They were infused with AAV8-TBG-Cre $\left(2.5 \times 10^{\wedge} 11 \mathrm{vg} / \mathrm{mouse}\right)$ through tail vein injection at 6.5 weeks of age. After 10 days, mice were treated with $\mathrm{Tm}(1 \mathrm{mg} / \mathrm{Kg})$ or vehicle (saline) through I.P. injection and were sacrificed for tissue collection after $17 \mathrm{~h}$.

\section{qRT-PCR and qPCR analyses.}

Total RNAs were extracted from isolated liver by RNeasy Mini Kit (Qiagen). cDNAs were synthesized by iScript cDNA Synthesis kit (Bio-Rad Laboratories, Inc). The relative mRNA levels were measured by qRT-PCR with iTaq Universal SYBR green Supermix (Bio-Rad Laboratories, Inc). All primers are listed in Suppl. Table 1.

\section{Immunofluorescence microscopy.}


Cells were plated on coverslips for overnight and fixed with 4\%PFA. Cells and Sections were stained with the following antibodies; FLAG (M2, Sigma), $\alpha$-PDIA6 (18233-1-AP, Proteintech), and DAPI (Fisher Scientific). For secondary antibodies we used: Alexa Fluor 488 goat $\alpha$-rabbit lgG, Alexa Fluor 594 goat $\alpha$-mouse IgG, anti-bodies (Invitrogen). Images were taken by a Zeiss LSM 710 confocal microscope with a $20 \mathrm{X}$ and $63 \mathrm{X}$ objective lenses. Scale bars are indicated in the figures.

\section{Western blot analyses.}

All Western blots were performed separating proteins by SDS-PAGE on a $5-15 \%$ gradient polyacrylamide gel for transfer onto nitrocellulose membranes, followed by blocking with Licor Blocking solution and incubation with primary and fluorescent-labeled secondary antibodies (Licor). The immune-fluorescent signals were captured using a Licor scanner. The key primary antibodies used in this study were as follows: Flag (M2, Sigma), BiP(3177, CST), KDEL (SC58774, SCBT), PDIA4 (14712-1-AP, Proteintech), PDIA6 (18233-1-AP, Proteintech), $\beta$-Actin (8H10D10, CST).

\section{RESULTS}

\section{Generation of BiP-FLAG mice.}

BiP-FLAG conditional knock-in mice were generated by targeting exon9 CDS region and flanking of with LoxP sites via gene targeting in Bruce4 C57BL/6 embryonic stem (ES) cells (14). Genetargeted ES cell clones were identified, and cells then injected into goGermline blastocysts (15, 16). Male chimeric mice were bred with Flp female mice to delete the Neo cassette and establish heterozygous germline offspring on a C57BL/6 background (Figure 1A). TaqMan $®$ copy number assay was used to genotype the offspring (Figure 1B). A total of 17 pups was born from three litters, including $10 \mathrm{WT}$ and $7 \mathrm{WT} /$ conKI (41\% observed vs. 50\% expected). All of these pups grew normally and appeared healthy. No difference in body weights between genotypes was observed (Suppl. Figure 1).

To test if the Cre-induced Hspa5-FLAG allele can deplete the endogenous Hspa5 allele, we injected AAV-Cre by intravenous injection into mouse tails as described for the in vivo experiments. To test measure mRNA expression for the endogenous and targeted Hspa5 allele, we performed qRT-PCR with primers directed at the targeted region, including crossing FLAG region and within the FLAG region. With AAV-Cre induced LoxP deletion in liver, WT mice 
demonstrated an $\sim 2$-fold increased expression compared to the BiP-FLAG-Het mice with primer Hspa5-exon 9 that identifies the WT allele, as expected. While the other Hspa5 primer that does not target the FLAG region did not show significant difference between the WT and knockin mice. The primer targeting the FLAG sequence was only observed upon amplification in BiPFLAG-Het mice. This confirmed the FLAG knock-in into the Hspa5 locus at exon 9 (Figure 1C).

\section{BiP-FLAG expression ex vivo in primary hepatocytes and skin fibroblasts isolated from BiP-FLAG heterozygous (Het) mice.}

To activate BiP-FLAG expression ex vivo, we isolated primary hepatocytes and skin fibroblasts from a heterozygous BiP-FLAG mouse and transduced them with Ad-Cre to induce Cre-mediated deletion of the floxed Hspa5 segment. At $24 \mathrm{~h}$ after Ad-Cre-transduction, approximately $90 \%$ of the BiP-FLAG-Het hepatocytes and fibroblasts were positive for FLAG immunofluorescence (Suppl. Figure 2).

In hepatocytes, western blot analysis detected BiP-FLAG migrating slightly above endogenous $\mathrm{BiP}$ in the BiP-FLAG-Het hepatocytes as early as $22 \mathrm{~h}$ after Ad-Cre transduction (not shown). By 3 days after Ad-Cre transduction, the steady-state-level of BiP-FLAG was about $50 \%$ of the endogenous BiP under basal conditions but increased to a level similar to that of the endogenous BiP produced from the untargeted Hspa5 allele after Tm-treatment for $20 \mathrm{~h}$, based on western blotting analysis with a rabbit anti-BiP monoclonal antibody (Figure 2), suggesting that the 3xFLAG insertion into the BiP C-terminus does not alter Hspa5 expression in our mouse model. Significantly, very similar levels of total BiP were observed in the Ad- $\beta$ Gal- and Ad-Cretransduced BiP-FLAG-Het hepatocytes after $20 \mathrm{~h}$ Tm-treatment (Figure 2), suggesting that our genetic modification of Hspa5 did not alter the UPR response.

Unlike the primary hepatocytes, Ad-Cre activation of the BiP-FLAG knock-in locus in BiP-FLAGHet primary skin fibroblasts resulted in equal levels of endogenous BiP and BiP-FLAG (Figure 3). This difference between skin fibroblasts and primary hepatocytes may be explained by the fact that fibroblasts, but not hepatocytes, proliferate in vitro, leading to a dilution of the preexisting endogenous BiP in the fibroblasts. Significantly, the increase in endogenous BiP and BiP-FLAG were nearly identical in response to castanospermine (CST)- or Tm-treatment to activate the UPR (Figure 4).

\section{BiP-FLAG is localized to the ER.}


An essential question is whether tagging the C-terminus of BiP may alter its intracellular localization as the FLAG tag is adjacent to the KDEL ER retention signal. Immunofluorescence microscopy showed that BiP-FLAG colocalized with the ER localized PDIA6 both in Ad-Cretransduced BiP-FLAG-Het primary hepatocytes (Figure $5 \mathrm{~A}$ ) and skin fibroblasts (Figure 5B), importantly demonstrating that insertion of the 3xFLAG tag into BiP did not alter its cellular localization.

\section{Hepatocyte-specific Cre-expression in BiP-FLAG-Het mice demonstrates intact functional activities of BiP-FLAG in vivo.}

Together, the above findings show that BiP-FLAG knock-in did not alter the expression, localization or the functional activity of the endogenous or the modified Hspa5 alleles. To confirm these findings in vivo and to explore the feasibility for hepatocyte-specific BiP-FLAG knock-in, we infused AAV8-TBG-Cre into 4 BiP-FLAG-Het mice and 4 WT littermates to express Cre selectively in hepatocytes. The TBG promoter is a hybrid promoter comprised of the human thyroxine-binding globulin promoter and microglobin/bikunin enhancer that is specifically expressed in hepatocytes. These mice were treated with $\mathrm{Tm}(1 \mathrm{mg} / \mathrm{Kg}$ ) or vehicle (saline) at day 10 after AAV8-infusion for $17 \mathrm{~h}$. Cre-mediated activation of BiP-FLAG in hepatocytes of BiP-FLAG-Het mice did not alter plasma or hepatic lipid levels and did not alter liver morphology in the absence or presence of Tm-treatment (Figure 6ABC, Suppl. Figure 3). In addition, BiP-FLAG was detected in nearly all hepatocytes in the livers of both AAV8-TBG-Cre-infused BiP-FLAG-Het mice.

Like the Ad-Cre-transduced BiP-FLAG-Het skin fibroblasts, there were equivalent levels endogenous BiP and BiP-FLAG in the livers of the AAV8-TBG-Cre-treated BiP-FLAG-Het mice with or without Tm-treatment (Figure 7). Importantly, BiP-FLAG knock-in did not alter expression of GRP94, PDIA4 and PDIA6 as well as BiP in the livers under basal or Tm-induced conditions (Figure 7). qRT-PCR assay demonstrated that activation of the Hspa5-FLAG allele did not alter expression of key UPR genes under basal or induced conditions (Figure 8).

Finally, to confirm the ability of anti-FLAG antibody to immunoprecipitate (IP) BiP-FLAG synthesized in vivo, we performed FLAG-IP assays of liver lysates prepared from the BiP-FLAGHet mice. A mouse anti-FLAG antibody completely depleted BiP-FLAG from the IP supernatants of the AAV8-TBG-Cre-treated BiP-FLAG-Het liver lysates (Figure 9), demonstrating a high efficiency for BiP-FLAG pulldown. While more work is necessary to understand the nature and composition of the proteins pulled down with BiP-FLAG, the finding that a significant amount of 
endogenous BiP was pulled down with BiP-FLAG from the liver lysates of the AAV8-TBG-infected BiP-FLAG-Het mice, especially those with Tm-treatment (Figure 9), indicates that BiP-FLAG was pulled down as protein complexes.

\section{DISCUSSION}

We have demonstrated successful targeting of the endogenous Hspa5 locus in mice. The phenotypic characterization identified no defect in hepatocyte function, ER function or BiP-FLAG localization to the ER. We believe that the epitope-tagged Hspa5 locus will stimulate studies on BiP client specificity, function, and role in protein folding that was not previously possible. For example, FLAG IP of BiP-FLAG will permit a new ability to answer fundamental questions: 1) Do different proteins misfold and bind BiP in healthy vs. diseased cells?; 2) Do all inducers of ER stress in generate similar protein misfolding consequences or are there differences depending on the degree or type of ER stress, or cell type?; 3) What proteins exist in different complexes that contain $\mathrm{BiP}$ ?; 4) What are the kinetics of misfolded protein interaction and release from BiP?; and 5) How do BiP interactions impact general ER processes? The BiP-FLAG mice will enable for the first-time delineation of folding pathways for any specific protein in vivo.

\section{ACKNOWLEDGEMENTS}

R.J.K. is supported by NIH grants R01DK113171, R24DK110973, R37DK042394, R01CA198103, R01AG062190 and the SBP NCI Cancer Center Grant P30 CA030199. R.J.K. is a member of the UCSD DRC (P30 DK063491) and Adjunct Professor in the Department of Pharmacology, UCSD.

\section{REFERENCES}

1. Hebert DN, Molinari M. In and out of the ER: protein folding, quality control, degradation, and related human diseases. Physiol Rev. 2007;87(4):1377-408. doi: 10.1152/physrev.00050.2006. PubMed PMID: 17928587.

2. Kaufman RJ. Orchestrating the unfolded protein response in health and disease. J Clin Invest. 2002;110(10):1389-98. doi: 10.1172/JCI16886. PubMed PMID: 12438434; PMCID: PMC151822. 
3. Ron D, Walter P. Signal integration in the endoplasmic reticulum unfolded protein response. Nat Rev Mol Cell Biol. 2007;8(7):519-29. doi: 10.1038/nrm2199. PubMed PMID: 17565364.

4. Haas IG, Wabl M. Immunoglobulin heavy chain binding protein. Nature. 1983;306(5941):387-9. doi: 10.1038/306387a0. PubMed PMID: 6417546.

5. Munro S, Pelham HR. An Hsp70-like protein in the ER: identity with the 78 kd glucoseregulated protein and immunoglobulin heavy chain binding protein. Cell. 1986;46(2):291-300. doi: 10.1016/0092-8674(86)90746-4. PubMed PMID: 3087629.

6. Bertolotti A, Zhang Y, Hendershot LM, Harding HP, Ron D. Dynamic interaction of BiP and ER stress transducers in the unfolded-protein response. Nat Cell Biol. 2000;2(6):326-32. doi: 10.1038/35014014. PubMed PMID: 10854322.

7. Dorner AJ, Bole DG, Kaufman RJ. The relationship of $\mathrm{N}$-linked glycosylation and heavy chain-binding protein association with the secretion of glycoproteins. J Cell Biol. 1987;105(6 Pt 1):2665-74. Epub 1987/12/01. doi: 10.1083/jcb.105.6.2665. PubMed PMID: 3121636; PMCID: PMC2114744.

8. Kozutsumi Y, Segal M, Normington K, Gething MJ, Sambrook J. The presence of malfolded proteins in the endoplasmic reticulum signals the induction of glucose-regulated proteins. Nature. 1988;332(6163):462-4. Epub 1988/03/31. doi: 10.1038/332462a0. PubMed PMID: 3352747.

9. Dorner AJ, Wasley LC, Kaufman RJ. Increased synthesis of secreted proteins induces expression of glucose-regulated proteins in butyrate-treated Chinese hamster ovary cells. J Biol Chem. 1989;264(34):20602-7. PubMed PMID: 2511206.

10. Dorner AJ, Wasley LC, Kaufman RJ. Overexpression of GRP78 mitigates stress induction of glucose regulated proteins and blocks secretion of selective proteins in Chinese hamster ovary cells. EMBO J. 1992;11(4):1563-71. PubMed PMID: 1373378; PMCID: PMC556605.

11. Ng DT, Watowich SS, Lamb RA. Analysis in vivo of GRP78-BiP/substrate interactions and their role in induction of the GRP78-BiP gene. Mol Biol Cell. 1992;3(2):143-55. Epub 1992/02/01. doi: 10.1091/mbc.3.2.143. PubMed PMID: 1550958; PMCID: PMC275514. 
12. Scheuner D, Vander Mierde D, Song B, Flamez D, Creemers JW, Tsukamoto K, Ribick M, Schuit FC, Kaufman RJ. Control of mRNA translation preserves endoplasmic reticulum function in beta cells and maintains glucose homeostasis. Nat Med. 2005;11(7):757-64. doi: 10.1038/nm1259. PubMed PMID: 15980866.

13. Hidvegi T, Schmidt BZ, Hale P, Perlmutter DH. Accumulation of mutant alpha1-antitrypsin $Z$ in the endoplasmic reticulum activates caspases-4 and -12, NFkappaB, and BAP31 but not the unfolded protein response. J Biol Chem. 2005;280(47):39002-15. Epub 2005/09/27. doi: 10.1074/jbc.M508652200. PubMed PMID: 16183649.

14. Kontgen F, Suss G, Stewart C, Steinmetz M, Bluethmann H. Targeted disruption of the MHC class II Aa gene in C57BL/6 mice. Int Immunol. 1993;5(8):957-64. Epub 1993/08/01. doi: 10.1093/intimm/5.8.957. PubMed PMID: 8398989.

15. Koentgen F, Lin J, Katidou M, Chang I, Khan M, Watts J, Mombaerts P. Exclusive transmission of the embryonic stem cell-derived genome through the mouse germline. Genesis. 2016;54(6):326-33. Epub 2016/03/26. doi: 10.1002/dvg.22938. PubMed PMID: 27012318; PMCID: PMC5084746.

16. Zhou H, Zeng Z, Koentgen F, Khan M, Mombaerts P. The testicular soma of Tsc22d3 knockout mice supports spermatogenesis and germline transmission from spermatogonial stem cell lines upon transplantation. Genesis. 2019;57(6):e23295. Epub 2019/04/20. doi: 10.1002/dvg.23295. PubMed PMID: 31001916; PMCID: PMC6617806.

17. Wang S, Chen Z, Lam V, Han J, Hassler J, Finck BN, Davidson NO, Kaufman RJ. IRE1alphaXBP1s induces PDI expression to increase MTP activity for hepatic VLDL assembly and lipid homeostasis. Cell Metab. 2012;16(4):473-86. Epub 2012/10/09. doi: 10.1016/j.cmet.2012.09.003. PubMed PMID: 23040069; PMCID: PMC3569089. 


\section{FIGURE LEGENDS}

Figure 1. Generation of BiP-FLAG-Het mice were generated by homologous recombination. A. Targeting strategy. The replacement vector contains a Neo-cassette (yellow) flanked by FRT sites (green) which, together with targeted WT exon 9-pA cassette, are flanked by LoxP sites (red) followed by duplicated exon 9 containing a 3xFLAG sequence(red) upstream from the KDEL motif. Using 5' and 3' homology arms the vector was used to target the Hspa5 locus of murine ES cells. The Neo-cassette was removed by Flp-mediated recombination. After Cre mediated recombination, exon 9-FLAG is expressed under control of the endogenous Hspa5 regulatory elements. Blue boxes denote exons. Lo5WT, Lo3WT and GoConK represent qPCR probes for the specified locus.

B. Copy number quantification. qPCR was performed to analyze BiP-FLAG-Het mice. qPCR reactions display genotyping from WT/WT-Flp/FIp, WT/conKI-WT/Flp (mice number, 2093_044_A048F), and WT/conKI-WT/FIp(mice number, 2093_044_A047F) mice. Primers are indicated in panel A. Lo5WT and LoWT3 identify the WT allele of Hspa5. goConK identifies the knock-in allele of Hspa5.

C. Targeted allele expression. Total RNA was extracted from BiP-FLAG-Het mice liver infected with AAV8-TBG-Cre. mRNA expression of Hspa5 was measured by qRT-PCR.

Figure 2. The BiP-FLAG allele is induced by ER stress in primary hepatocytes. Hepatocytes were isolated from a 6-wk old female BiP-FLAG-Het mouse, plated onto 24 well plates and infected with the indicated adenoviruses at $4 \mathrm{~h}$ after plating. After $48 \mathrm{~h}$, cells were treated with $\mathrm{Tm} 0.5 \mu \mathrm{g} / \mathrm{ml}$ or vehicle for $20 \mathrm{~h}$ and then harvested for Western blot analysis.

Figure 3. BiP-FLAG is activated by Ad-Cre infection in BiP-FLAG primary fibroblasts. Skin fibroblasts were isolated from a 6-wk old female BiP-FLAG-Het mouse and plated onto 24 well plates, infected with the indicated adenoviruses after the first passage and harvested for Western blot analysis.

Figure 4. BiP-FLAG is induced in BiP-FLAG primary fibroblasts in response to ER stress. Skin fibroblasts were isolated from a 6-wk old female BiP-FLAG-Het mouse, plated onto 24 well plates, infected with the indicated adenoviruses after the first passage. After Ad-infection, cells were treated with $\mathrm{Tm} 0.5 \mu \mathrm{g} / \mathrm{ml}$, CST $20 \mu \mathrm{M}$ or vehicle for $22 \mathrm{~h}$ and harvested for Western blot analysis. 
Figure 5. BiP-FLAG is localized to the ER lumen. A. Hepatocytes were isolated from a 6-wk old female BiP-FLAG-Het mouse, plated onto 6 well plates and infected with the indicated adenoviruses at $4 \mathrm{~h}$ after plating. After 4 days, cells were fixed with formalin and stained with antiFLAG antibody, anti-PDIA6 antibody and DAPI. Images were captured by a 63 oil lens from each group by confocal microscopy. Scale bar, $20 \mu \mathrm{m}$. B. Fibroblasts were isolated from a 6 -wk old female BiP-FLAG-Het mouse, plated onto 6 well plates and infected with the indicated adenoviruses at 7 days after plating. At $24 \mathrm{~h}$ after Ad-infection, cells were fixed with formalin, stained with antibodies for FLAG or PDIA6 and for DAPI. Images were captured by a 63 oil lens from each group by confocal microscopy. Scale bar, $20 \mu \mathrm{m}$.

Figure 6. AAV8-TBG-Cre-treatment of BiP-FLAG-Het mice does not alter plasma or hepatic lipid content. Plasma cholesterol and triglyceride levels and hepatic triglyceride content of AAV8TBG-Cre-treated Hspa5 wildtype (WT) and BiP-FLAG-Het (Het) mice at $17 \mathrm{~h}$ after injection with vehicle (PBS) or Tm were measured. Each data point represents one individual mouse.

Figure 7. BiP-FLAG induction in hepatocytes by ER stress in vivo. After infection with AAV8TBG-Cre or control virus for 10 days, mice were treated with $\mathrm{Tm}(1 \mathrm{mg} / \mathrm{Kg}$ ) or vehicle (saline). After $17 \mathrm{~h}$, liver tissues were collected immediately after sacrifice and for lysis in RIPA buffer. Each lane in the Western blot represents an individual mouse.

Figure 8. Hepatocyte-specific BiP-FLAG knock-in does not alter expression of key UPR genes in the liver. Total RNAs were isolated from liver samples collected as described in Figure 7 and subjected to qRT-PCR analysis to measure mRNA levels for the indicated genes normalized to $18 \mathrm{~S}$ rRNA.

Figure 9. BiP-FLAG is efficiently pulled down from AAV8-TBG-Cre/BiP-FLAG-Het liver lysates using murine anti-Flag (M2) agarose. Liver lysates prepared as described in Figure 7 were subjected to IP using anti-FLAG (m2)-coupled to agarose. Western blotting was performed using a rabbit anti-BiP monoclonal antibody $(3177, \mathrm{CST})$ as a primary antibody. 


\section{Supplemental Figure Legends}

\section{Suppl. Figure 1. Body weight of WT and BiP-FLAG mice.}

Body weights were measured at 6-8 weeks of age from WT and BiP-FLAG knock-in mice. WT, males $\mathrm{N}=6$, females $\mathrm{N}=4$. BiP-FLAG, males $\mathrm{N}=2$, females $\mathrm{N}=5$.

\section{Suppl. Figure 2. AAV infection efficiency in primary hepatocytes and fibroblasts.}

A. Hepatocytes were isolated from a 6 -wk old female BiP-FLAG-Het mouse, plated onto 6 well plates and infected with the indicated adenoviruses at $4 \mathrm{~h}$ after plating. After $24 \mathrm{~h}$, cells were fixed with formalin and stained with anti-FLAG antibody and DAPI. B. Fibroblasts were isolated from a 6-wk old female BiP-FLAG-Het mouse, plated onto 6 well plates and infected with the indicated adenoviruses at 7 days after plating. At $24 \mathrm{~h}$ after Ad-infection, cells were fixed with formalin and staining with anti-FLAG antibody and DAPI. Four Images were randomly captured from each group by Zeiss 710 confocal microscopy. Scale bar, $50 \mu \mathrm{m}$. Quantification was performed by Image J. Quantification of the ratio of FLAG-positive cells to DAPI positive cells is shown in each graph (right).

\section{Suppl. Figure 3. Liver histology of AAV-TBG-Cre infected BiP-FLAG-Het mice.}

A. Morphology of hepatocytes based on H\&E stained liver sections of experimental mice. B. Pathohistological analysis and morphology of hepatocytes based on Oil Red $O$ stained liver sections of experimental mice. Experiments were performed with 2 mice in each group. Stained sections were scaned by Aperio, Leica Biosystems. The scale bar is $200 \mu \mathrm{m}$. 


\section{Figure 1}

A

Wild type Locus of the Hspa5 Gene

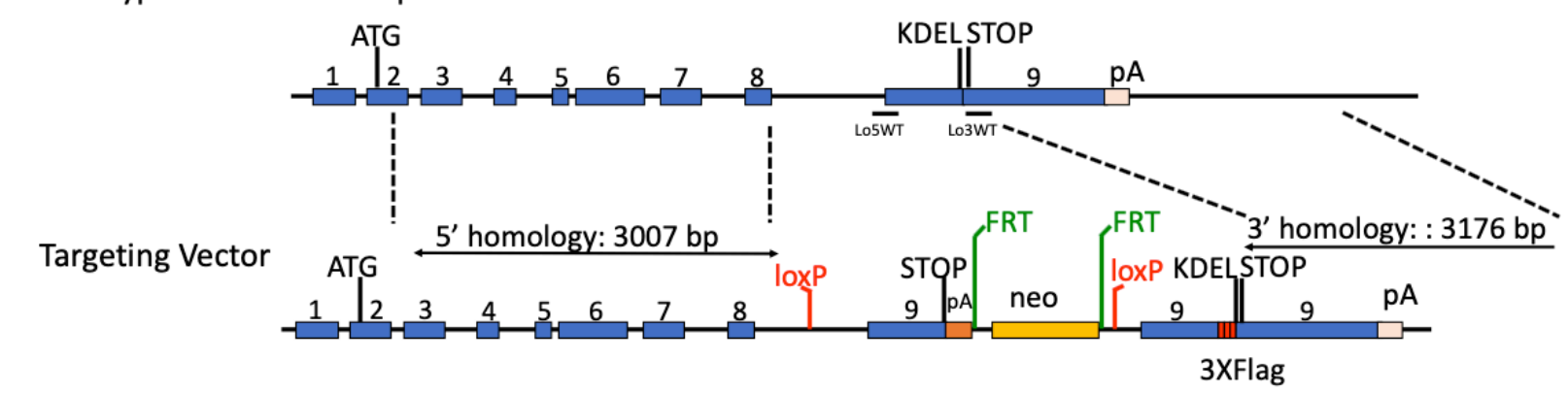

B
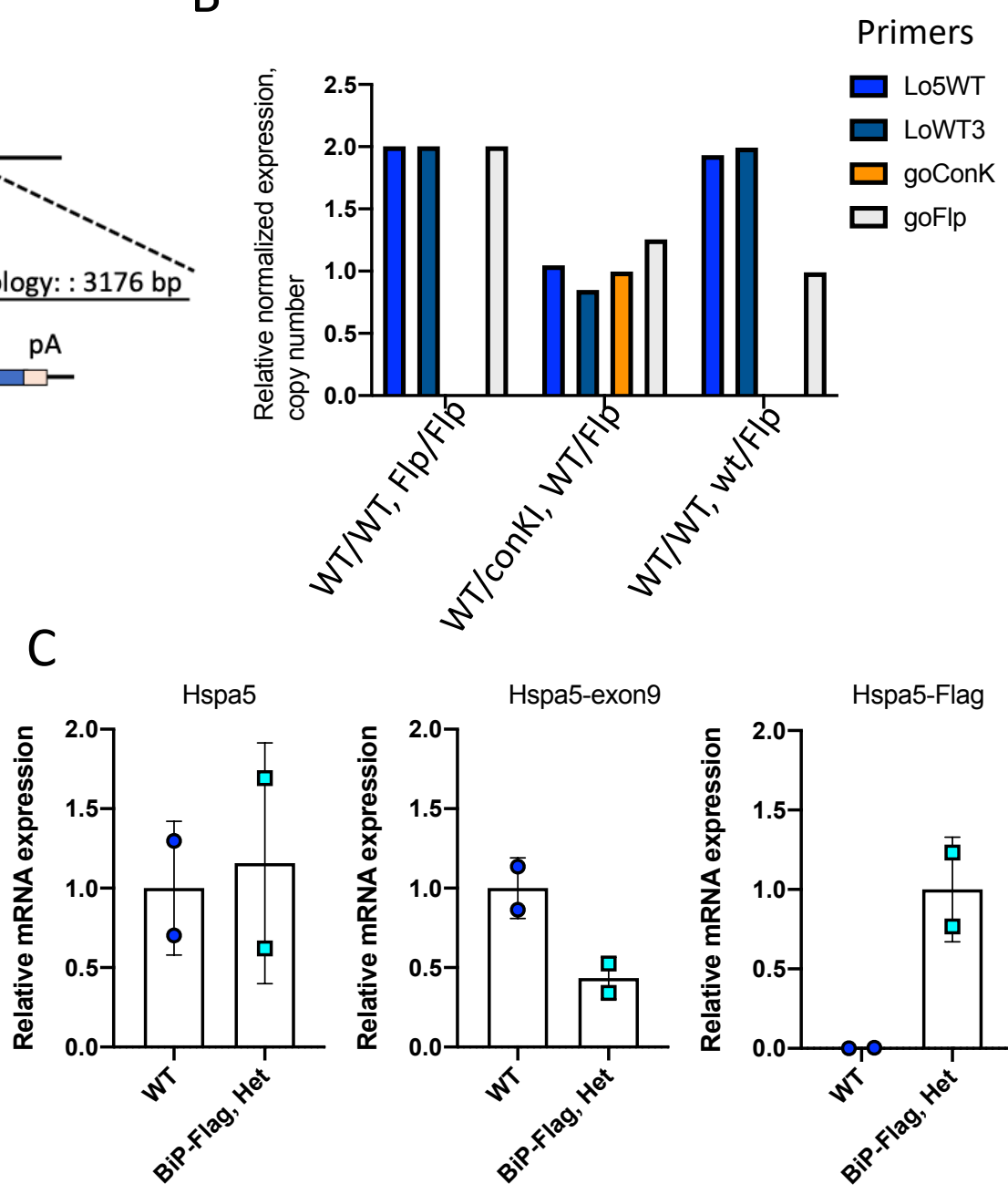
Figure 2

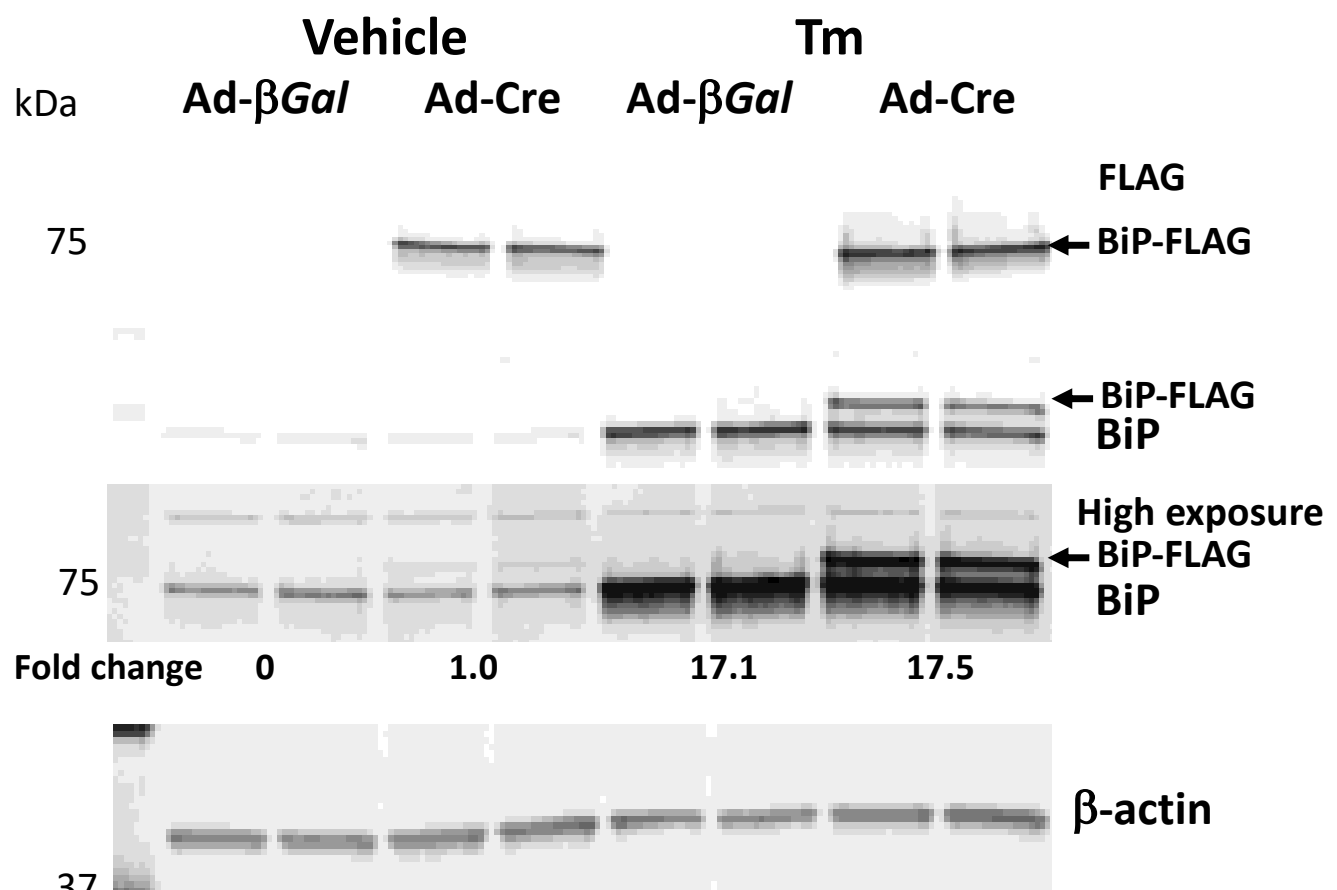

37 


\section{Figure 3}

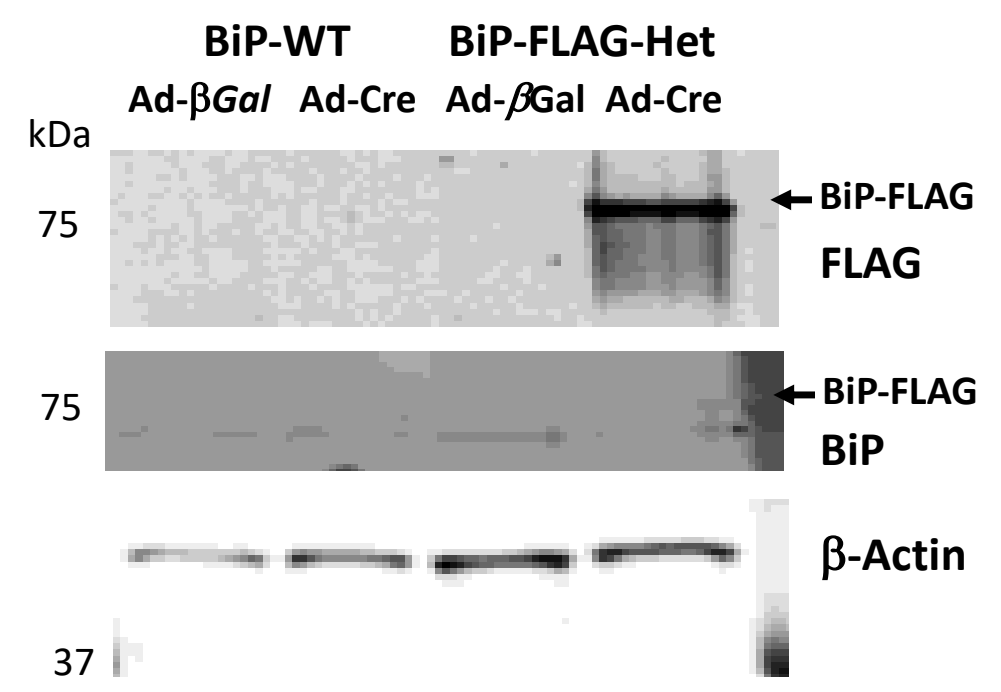


Figure 4

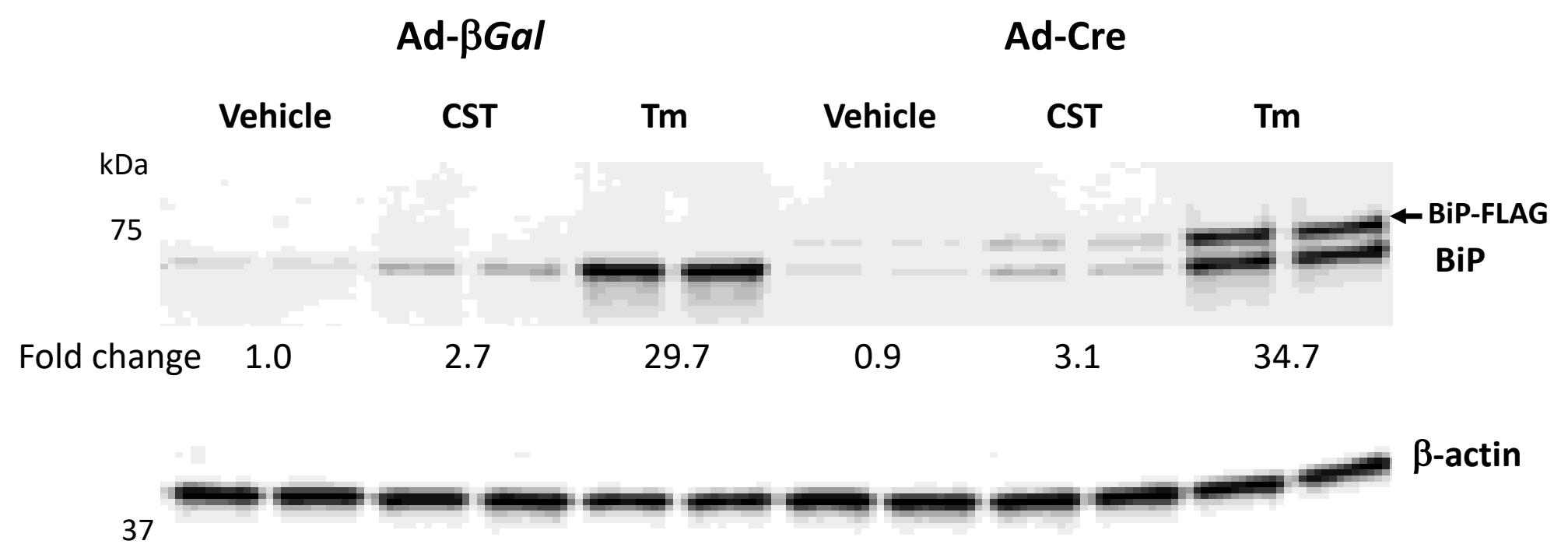


Figure 5
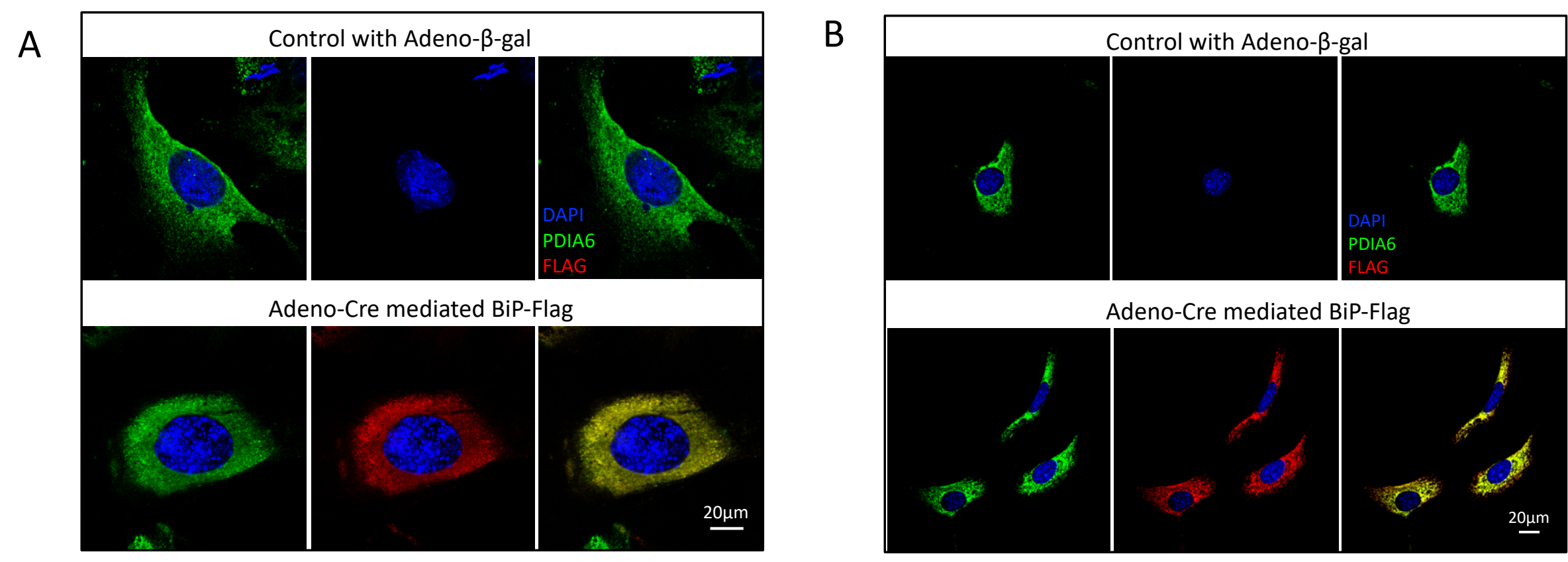
Figure 6
A. Plasma Cholesterol
B. Plasma Triglycerides
C. Liver Triglycerides
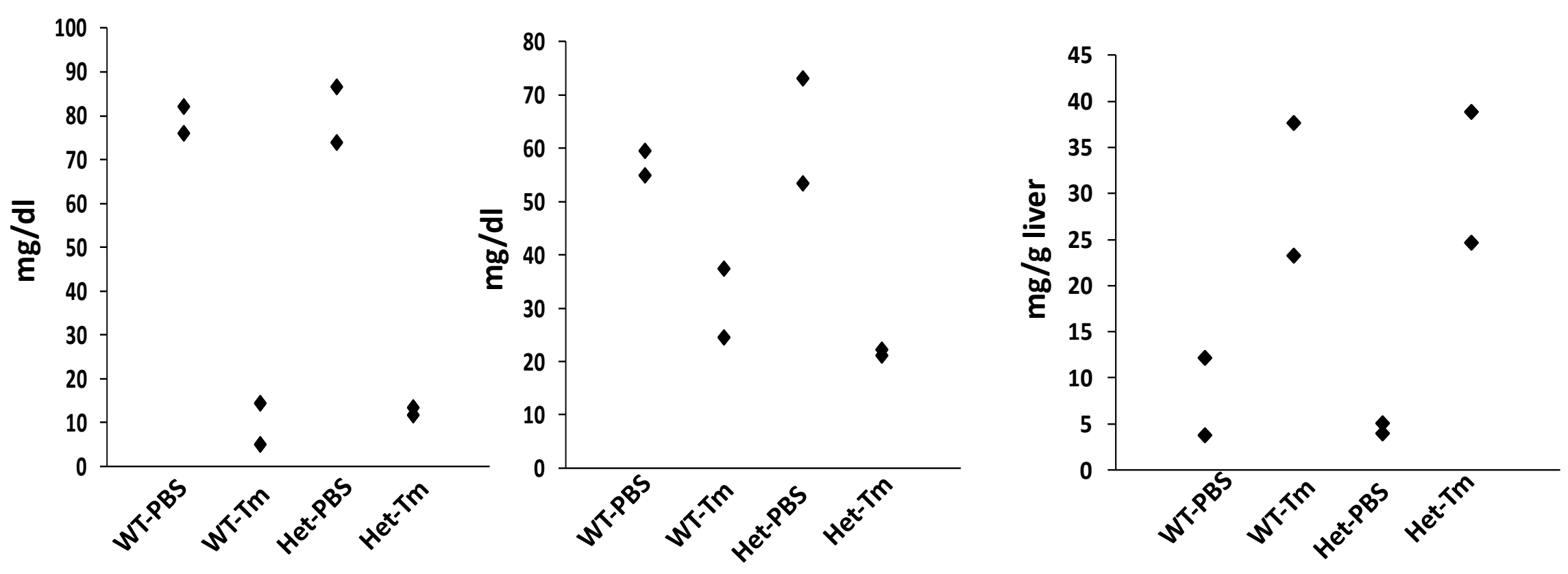


\section{Figure 7}

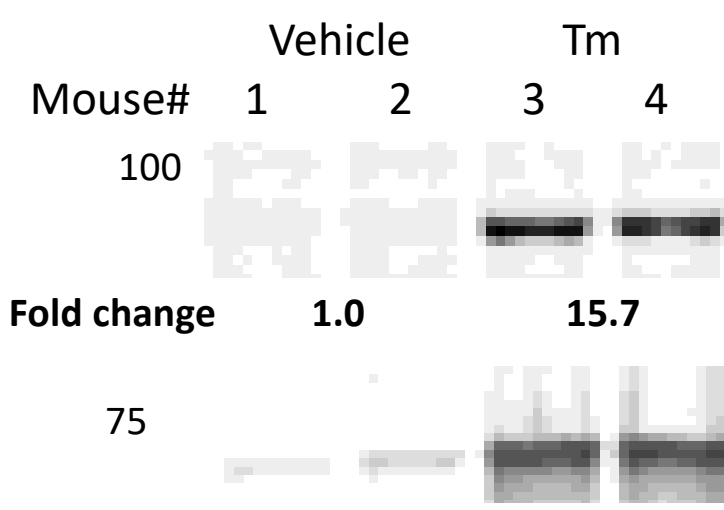

Fold change

1.0

31.4

75

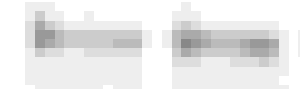

Fold change

1

2.9
50

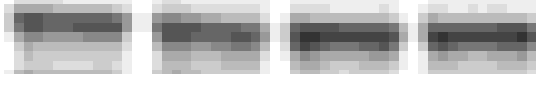

Fold change

1

1.2

0.9

1.1

25

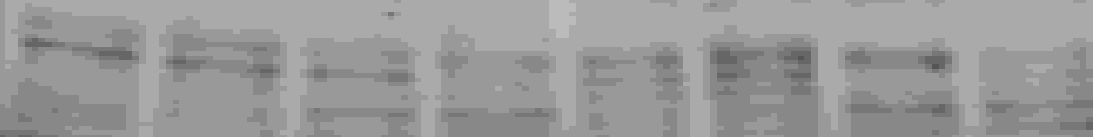

37

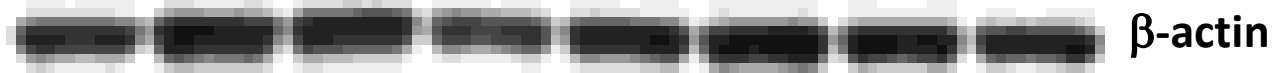

$\operatorname{TRAP} \alpha$

$\beta$-actin 
Figure 8

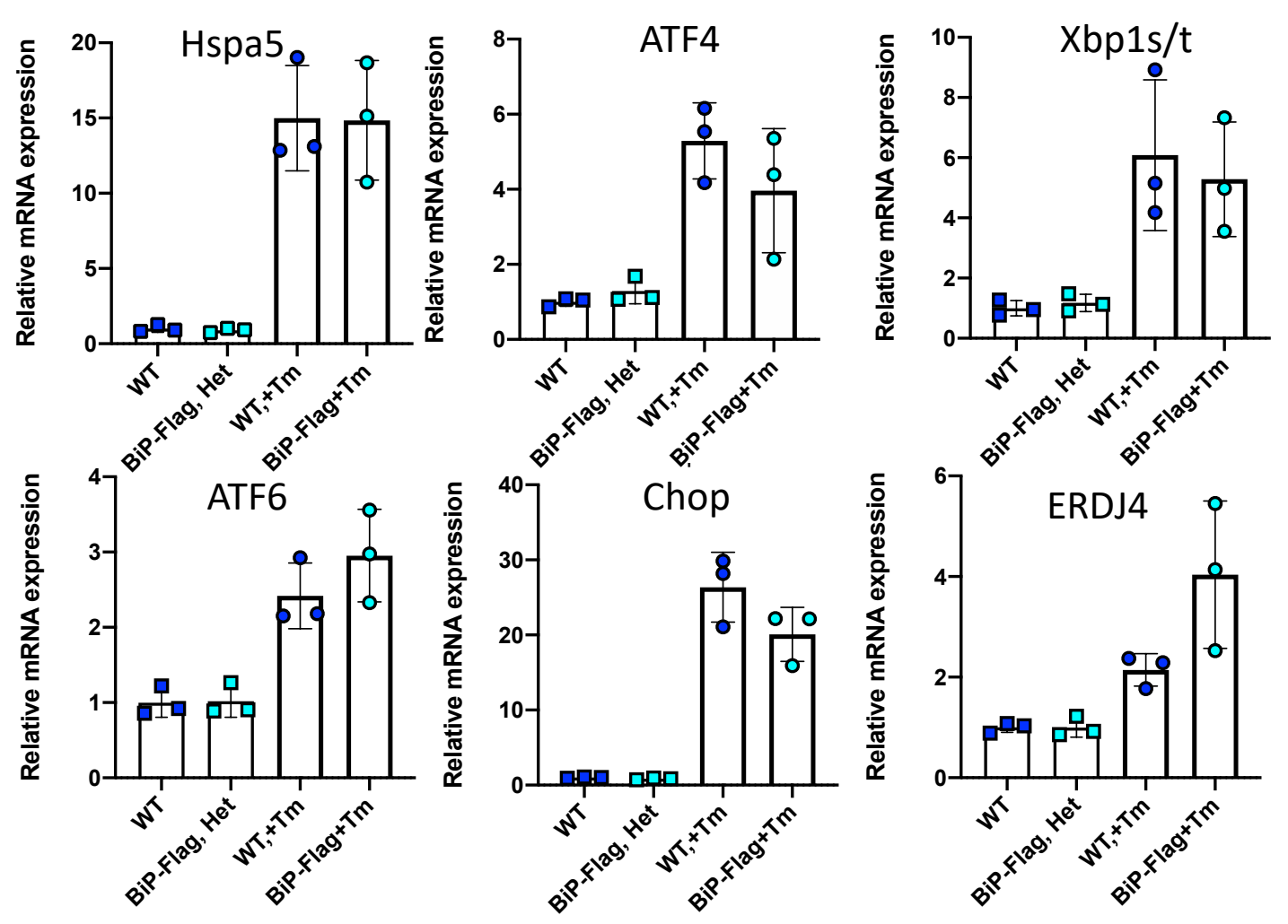


Figure 9

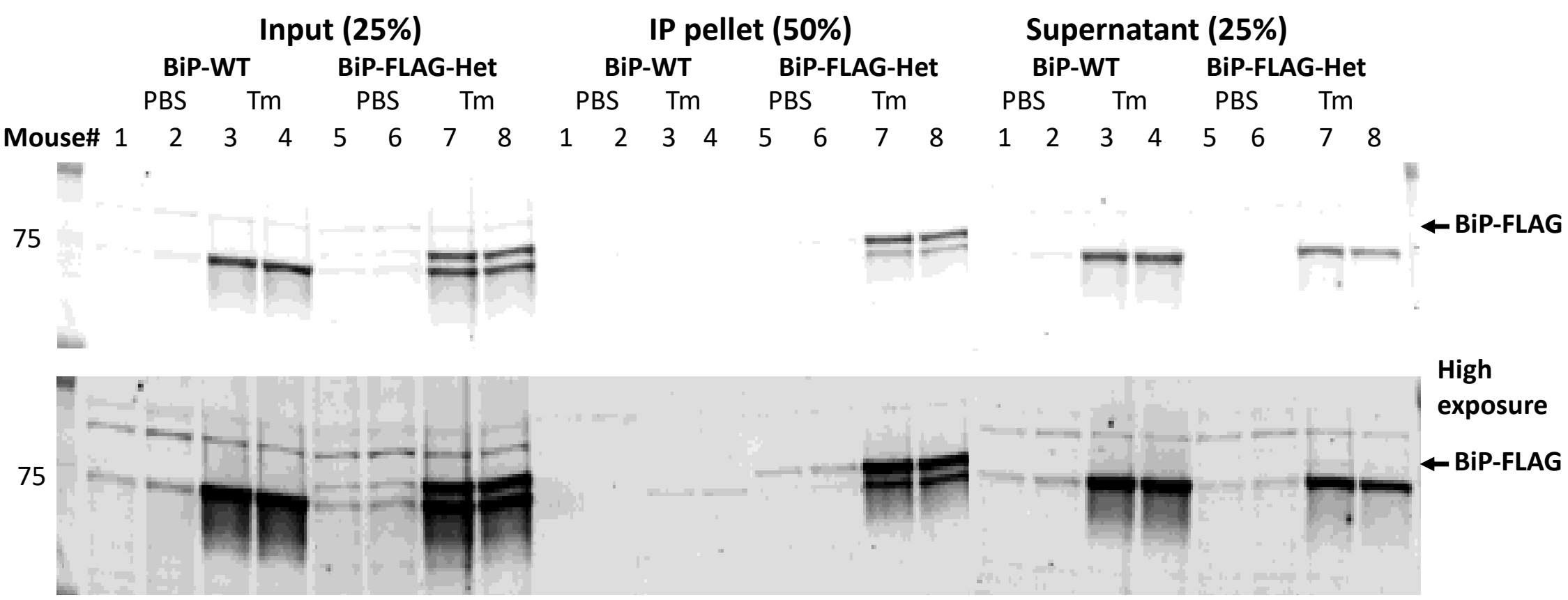

$\beta$-actin

37

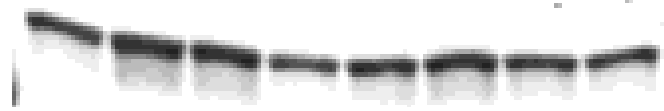




\section{Suppl. Table 1}

\section{Primers used}

\begin{tabular}{|c|c|c|}
\hline Name & Sequence & Used for \\
\hline P2093_41 & CTAACCTATTCCTGGTAAGTGGTATCCG & Targeting vector construction \\
\hline P2093_51 & TAAGCATTGGTAAGACGTCAAGCCCCTCTGACCTTGTATTAC & Targeting vector construction \\
\hline P2093_44 & TAAGCATTGGTAAGCGGCCGCGTGCACTGATCTGCTAGAGCTG & Targeting vector construction \\
\hline P2093_54 & CTAATGAACACAGAAGGGGAGGTTTATG & Targeting vector construction \\
\hline 2093_Lo5WT_F & GAGGGGCTTACAATGCTTTG & $S^{\prime}$ end Hspa5 wt allele validation \\
\hline 2093_Lo5WT_R & GGGTCGTTCACCTAGAGTAAG & 5 ' end Hspa5 wt allele validation \\
\hline 2093_Lo5WT_Probe & AAGAGCAGTAGCACCCAGTGAGTT & $S^{\prime}$ end Hspa5 wt allele validation \\
\hline 2093_LoWT3_F & AGATCAGTGCACCTACAA & 3' end Hspa5 wt allele validation \\
\hline 2093_LoWT3_R & CAGGATGCGGACATTGAA & 3' end Hspa5 wt allele validation \\
\hline 2093_LoWT3_Probe & AGCAAACTCTATGGAAGTGGAGGCC & 3' end Hspa5 wt allele validation \\
\hline 1638 goNoz_F & CTTCTTGACGAGTTCTTCTGAGG & Gain of Neo validation \\
\hline 1638 goNoz_R & AACAACAGATGGCTGGCA & Gain of Neo validation \\
\hline 1638 goNoz_Probe & TCAGCCTCGACTGTGCCTTCTAGT & Gain of Neo validation \\
\hline $1638 \_$goFlpOz_F & ATTGAGGAGTGGCAGCATATAG & Gain of Flp validation \\
\hline $1638 \_$goFlpOz_R & GGTAGTCTAGTACCTCCTGTGATA & Gain of Flp validation \\
\hline $1638 \_$goFlpOz_Probe & TGCTTCCTTCAGCACTACCCTTTAGC & Gain of Flp validation \\
\hline 2093_GoConK_F & GATTCAGTAGACCGCTGTTGG & $\begin{array}{l}\text { Gain of 2093_Teak conKI allele } \\
\text { validation }\end{array}$ \\
\hline 2093_GoConK_R & GCACTGGGATCCCTACATTAAC & $\begin{array}{l}\text { Gain of } 2093 \text { Teak conKI allele } \\
\text { validation }\end{array}$ \\
\hline 2093_GoConK_Probe & TGAAATTAAGAGCAGTAGCACCCAGTGA & $\begin{array}{l}\text { Gain of } 2093 \text { Teak conKI allele } \\
\text { validation }\end{array}$ \\
\hline 2093_GoK_F & TATCCTCCCAGGGAAAGA & $\begin{array}{l}\text { Gain of } 2093 \text { Teak conKI allele } \\
\text { validation }\end{array}$ \\
\hline 2093_GoK_R & AGCATTGTTCTAGACCGC & $\begin{array}{l}\text { Gain of } 2093 \text { Teak conKI allele } \\
\text { validation }\end{array}$ \\
\hline 2093_GoK_Probe & AAGCCCCTCTGACCTTGTATTAC & $\begin{array}{l}\text { Gain of } 2093 \text { Teak conKI allele } \\
\text { validation }\end{array}$ \\
\hline
\end{tabular}


Suppl. Figure 1

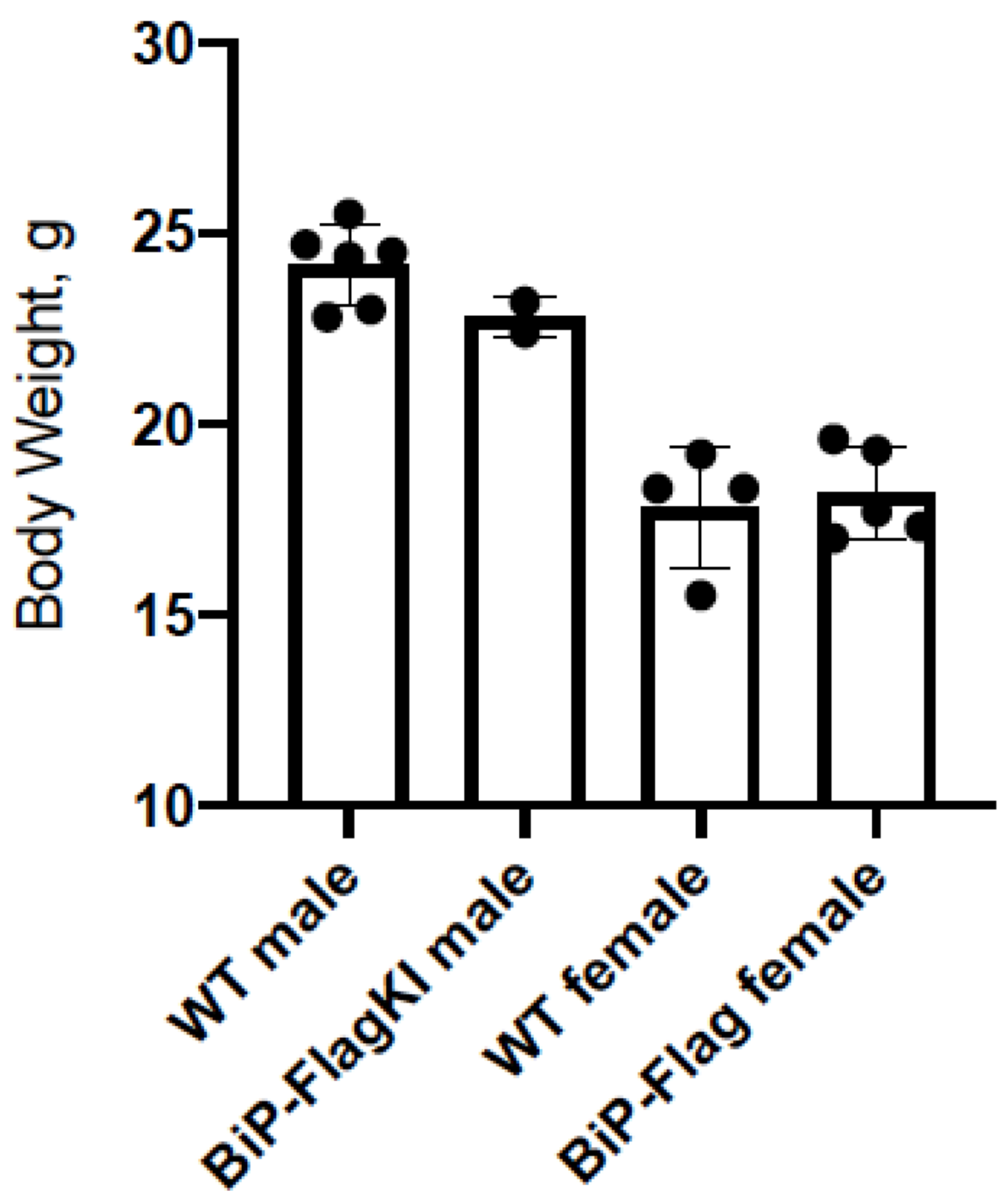




\section{Suppl. Figure 2}

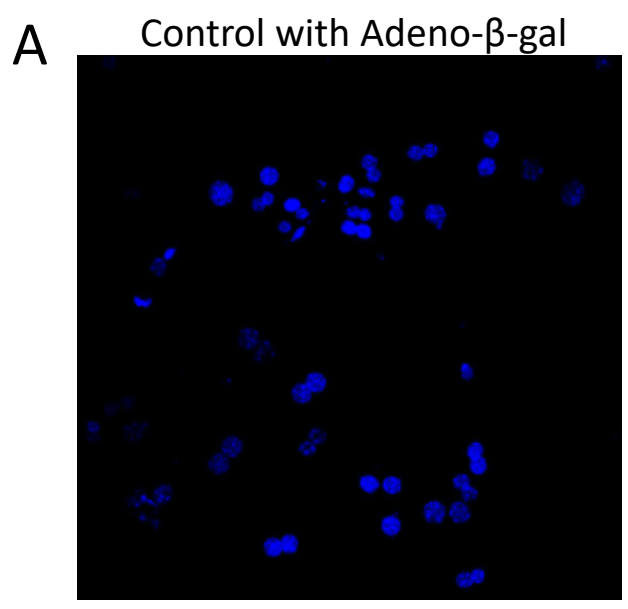

Adeno-Cre mediated BiP-Flag

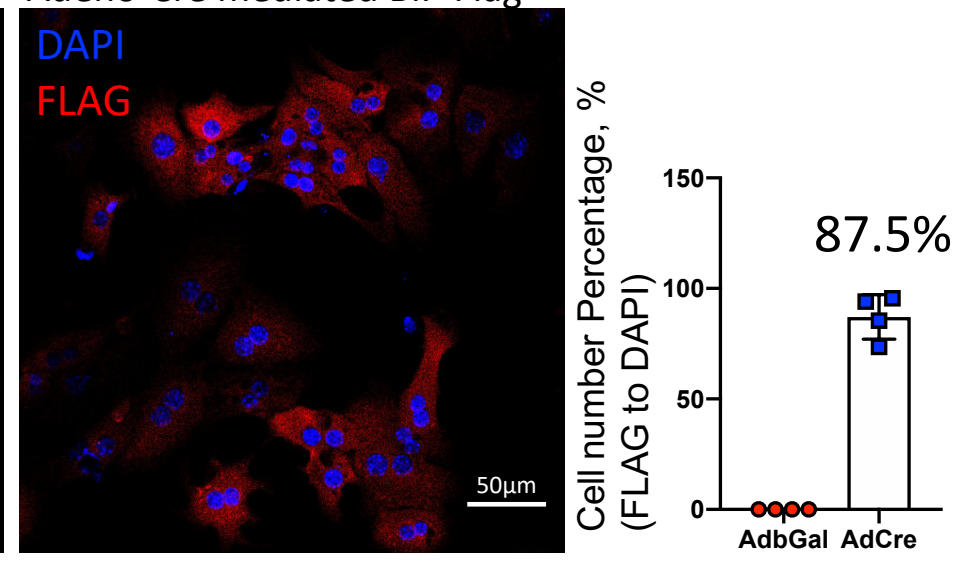

B Control with Adeno- $\beta$-gal

Adeno-Cre mediated BiP-Flag
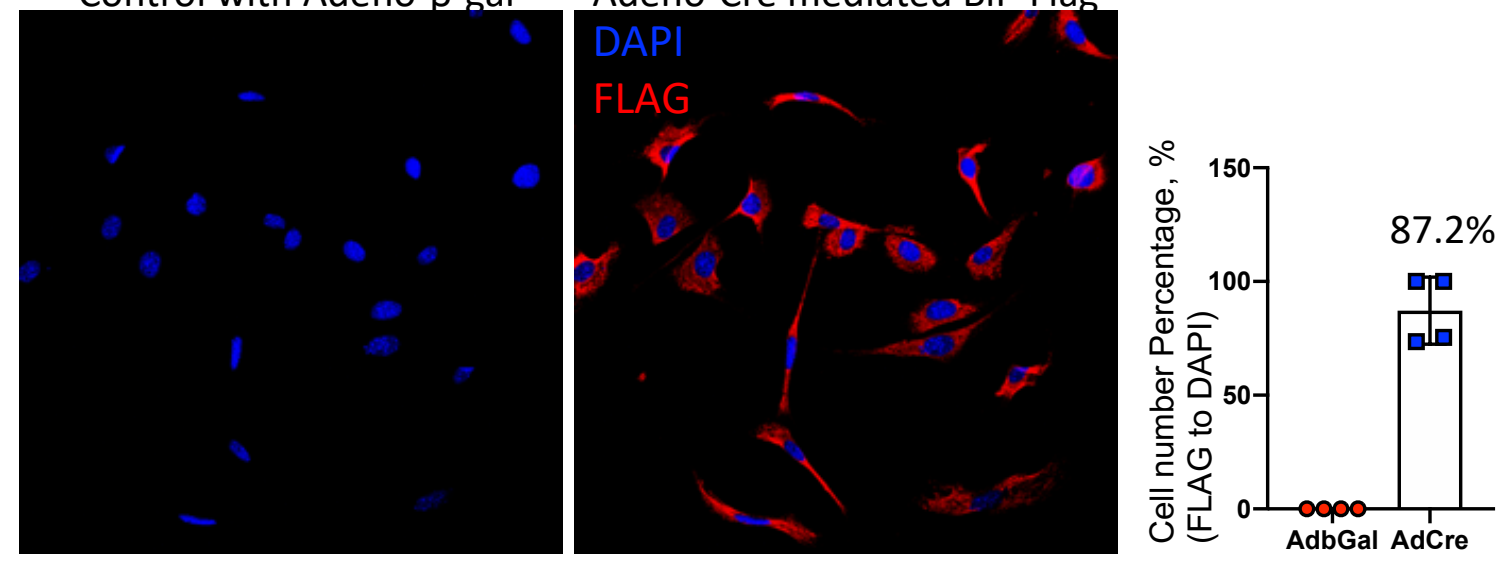


\section{Suppl. Figure 3}

A

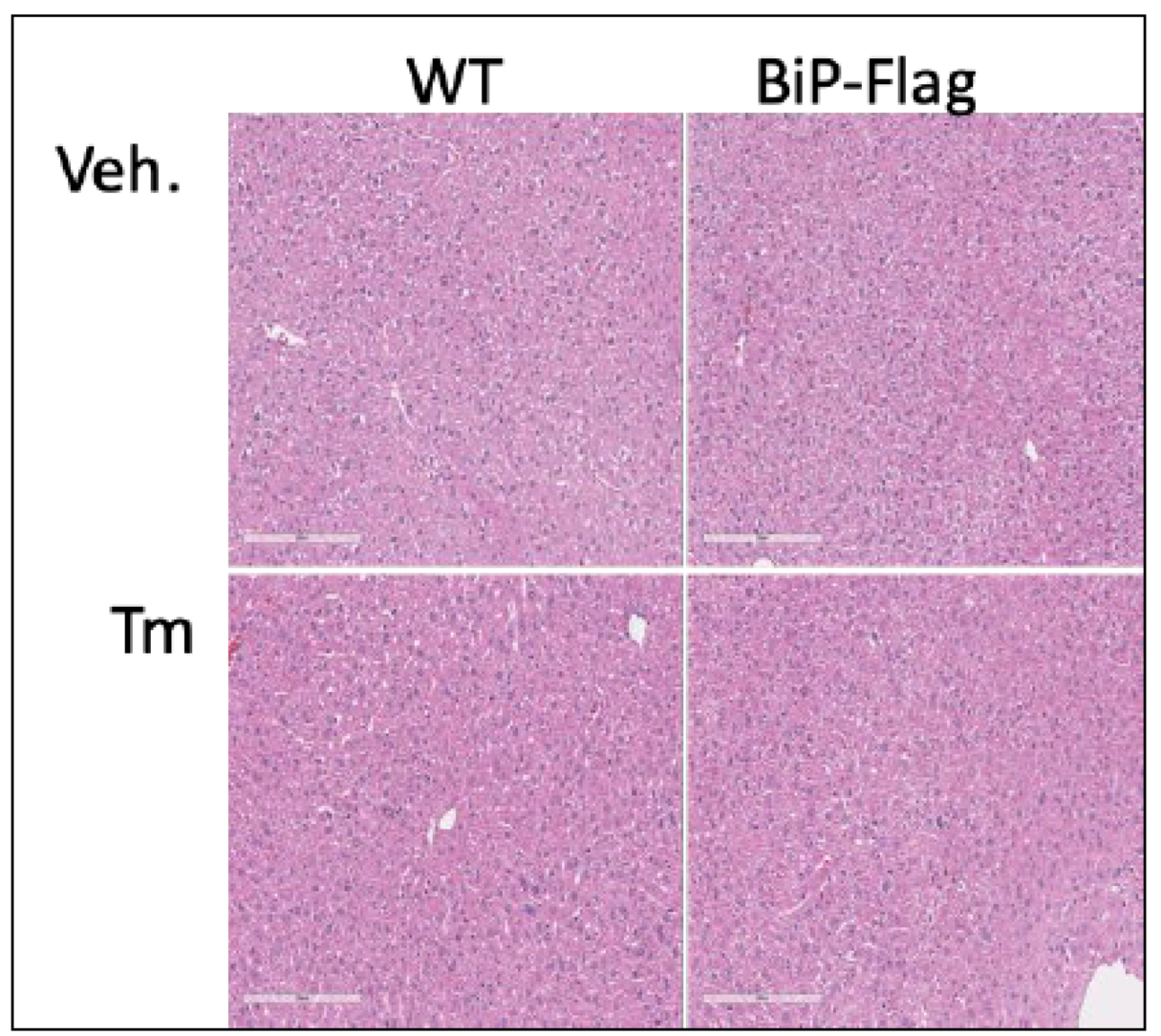

B

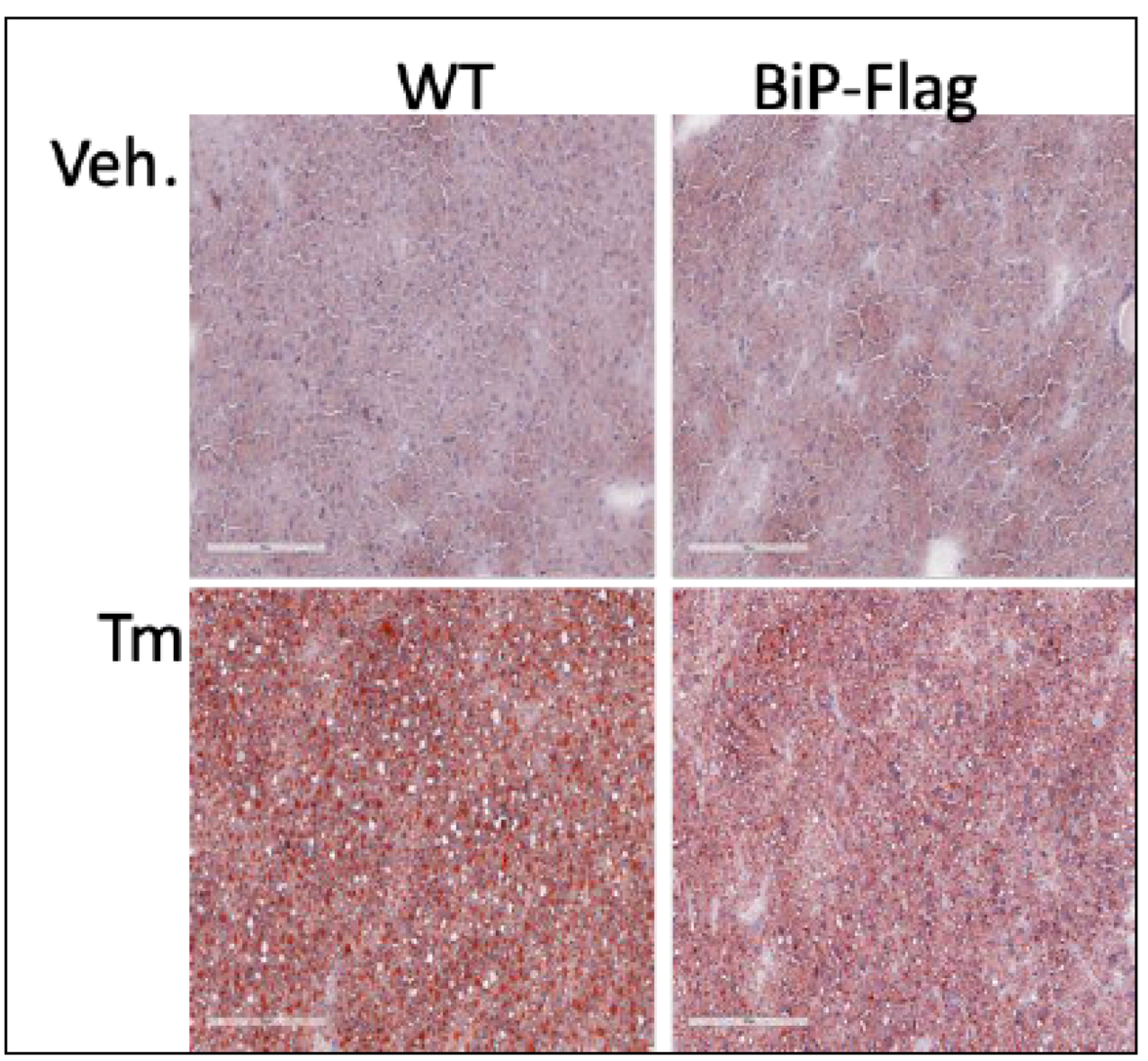

